\title{
Evaluación socio formativa en los procesos investigativos del programa de Pedagogía infantil
}

\section{Socio-educational evaluation in the research processes of the children's Pedagogy programme}

\author{
Fredy Andrés Cruz - Vega ${ }^{1}$ \\ ${ }^{1}$ Magister en Educación, Grupo de investigación: Pedagógica, Universidad de Pamplona, Colombia, \\ ORCID: https://orcid.org/0000-0002-1329-8948, E-mail: fredy.cruz@unipamplona.edu.co
}

Cómo citar: Cruz - Vega, F. A. (2019). Evaluación socio formativa en los procesos investigativos del programa de pedagogía infantil. Revista Científica Profundidad Construyendo Futuro, 10(10), 10-23. https://doi.org/10.22463/24221783.2523

Recibido: 08 de agosto de 2018 / Aprobado: 12 de septiembre de 2018

\section{Resumen}

El propósito del siguiente artículo es evidenciar de manera crítica y reflexiva la evaluación socio formativa del curso de proceso de investigación orientado en el programa de Licenciatura en Pedagogía, demostrar como es el proceso de formación desde una práctica profesional que se especializa en el conocimiento de las características propias del desarrollo multidimensional de los niños, favoreciendo la relación teoría-práctica, construidas y demostrables por el docente en la interacción interdisciplinaria del saber ser pedagogo. A su vez, como este es campo de reflexión sobre el futuro desempeño; permita formular proyectos de investigación que redunden en beneficio de la comunidad educativa, especialmente en la formación integral, inclusión social y la articulación a las políticas del estado como lo es el plan nacional de lectura y escritura. La metodología trabajada es de corte cualitativo, se enfatizó en el análisis de caso de una experiencia de evaluación dentro de esta asignatura permitiendo medir y demostrar como la articulación de la teoría con la realidad puede formar de manera eficiente a los licenciados.

Palabras clave: Desarrollo, Evaluación, Prácticas pedagógicas, Reflexivo, Socio formativo.

\begin{abstract}
The purpose of the following article is to demonstrate in a critical and reflective way the socio-formative evaluation of the course of the research process oriented in the Bachelor of Pedagogy program, to demonstrate how the training process is from a professional practice that specializes in the knowledge of the characteristics characteristic of the multidimensional development of children, favoring the theory-practice relationship, built and demonstrable by the teacher in the interdisciplinary interaction of knowing how to be a pedagogue. In turn, as this is a field of reflection on future performance; It allows the formulation of research projects that benefit the educational community, especially in comprehensive training, social inclusion, and articulation with State Policies such as the national plan for reading and writing. The Methodology worked is qualitative, emphasized in the case analysis of an evaluation experience within this subject allowing to measure and demonstrate how the articulation of theory with reality can efficiently train graduates.
\end{abstract}

Keywords: Development, Evaluation, Pedagogical practices, Reflective, Training partner. 


\section{Introducción}

La evaluación de competencias de acuerdo con la definición oficial de la Comisión Europea, competencia es la capacidad demostrada de utilizar conocimientos y destrezas. El conocimiento es el resultado de la asimilación de información que tiene lugar en el proceso de aprendizaje. La destreza es la habilidad para aplicar conocimientos y utilizar técnicas a fin de completar tareas y resolver problemas.

Con lo anterior, se puede demostrar que el concepto de competencia radica en función del aprendizaje (Tobón, 2001, 2002, 2013a), con ello se pude también afirmar que es un mecanismo útil de formación vial en la generación de nuevos aprendizajes en la educación y que permite a las instituciones evolucionar y demostrar cambios en la prestación del servicio educativo teniendo en cuenta la sociedad, los medios y las necesidades que se presentan para resaltar las habilidades de las personas.

Hoy en día, hay diversos enfoques para abordar la evaluación del aprendizaje, de manera precisa el modelo socio formativo que consiste en un enfoque surgido en Iberoamérica que tiene sus bases en el constructivismo social y la epistemología de la complejidad (Tobón, 2001), promoviendo la transformación de las prácticas de formación en diferentes entornos (educativos, organizacionales y sociales) mediante la adaptación de estrategias e instrumentos a los retos de la sociedad del conocimiento, como portafolios, listas de cotejo, escalas de estimación, análisis de casos y rúbricas entre otras (Tobón, 2013). Esta adaptación consiste en articular los niveles de desempeño que propone la socioformación, como también el enfoque de problemas y la transversalidad (Tobón, 2012, p. 360).

A raíz de las nuevas tendencias educativas los docentes deben estar en inmersas actualizaciones en cuanto al proceso de enseñanza y aprendizaje, es así como surge nuevas pautas o rutas para evaluar o medir los conocimientos no solo en tomar si son adquiridos de manera memorística si no como son interpretados por los educandos de esta manera; la evaluación socio formativa establece esa manera práctica de determinar cómo los conocimientos pueden ser una eficiente construcción de experiencias en el aula que hacen que tanto docentes como estudiantes determinen sus niveles de entendimiento y de saberes si necesidad de establecer una evaluación doctrinaria, esta también permite la retroalimentación de experiencias vividas en las practicas a lo largo de su formación (Tobón, 2001).

\section{Marco Teórico}

La evaluación socio formativa, es un nuevo enfoque de la evaluación que se centra en desarrollar y mejorar el talento de las personas para afrontar los retos de la sociedad del conocimiento mediante el abordaje de problemas del contexto y la colaboración. Se puede mencionar que la evaluación socio formativa es un proceso de diagnóstico, retroalimentación y apoyo continuo a las personas, equipos, organizaciones y comunidades para que aprendan a resolver problemas del contexto retadores, mejoren en su actuación y desarrollen el talento necesario para la sociedad del conocimiento, mediante la autoevaluación, evaluación y heteroevaluación, teniendo como base la elaboración de productos (evidencias) e indicadores (o instrumentos) que posibiliten la meta cognición, a través del trabajo 
colaborativo y el pensamiento complejo (Tobón, 2015).

Se puede deducir que la evaluación debe ser continua. Puede llevarse a cabo en entornos presenciales, semi-presenciales $\mathrm{o}$ virtuales. este proceso asegura que la evaluación en los estudiantes es más eficiente y pertinente pues comprende una mejor actitud por parte del docente en cuanto al dar un parte más estructurado de la calidad de enseñanza que quiere impartir y como este es recibido o adquirido por los estudiantes colocando el proceso de obtener saberes en un proceso más de auto conciencia profesional, dichos diagnósticos permiten también dar cuenta de que necesidades se pueden encontrar en el aula y enfatizar nuevas estrategias de enseñanza para que al finalizar la evaluación no se convierta en un sistema de medir calidad si no de retroalimentación y de construcción propia para el educando.

Las principales características de la evaluación, retoma algunos elementos de los otros enfoques. De la evaluación conductual recoge el énfasis en la retroalimentación oportuna para lograr las metas. De la evaluación constructivista sigue el abordaje de los saberes previos, la autoevaluación y la consideración de los intereses de los estudiantes. Sin embargo, se diferencia de estas dos perspectivas por su marcado énfasis en actuar ante los problemas del contexto, la colaboración continua, el trabajo con el proyecto ético de vida y la formación " $\mathrm{La}$ evaluación socio-formativa es el proceso de apoyo a los estudiantes para que desarrollen el talento mediante la retroalimentación continúa basada en la autoevaluación, coevaluación y hetero-evaluación con base en un problema del contexto y el análisis de evidencias, buscando el mejoramiento continuo" (Tobón, 2014).
Si bien es cierto la articulación de las experiencias que se viven a diario en el ejercicio de formación permiten que los educandos reflexionen de manera coherente $\mathrm{s}$ praxis permitiéndole ser más autoconsciente en la trasformación de las saberes y como estos influyen en la generación de nuevas alternativas educativas que favorecen sus procesos educativos con ello también es posible generar un conocimiento eficiente para el desarrollo de sus procesos practicas siendo este reflexivo y constructor de nuevas estrategias que le dan un actuar en el reconocimiento de las necesidades presentadas y así lograr una nueva praxis.

La incidencia de los procesos de la evaluación socio formativa en el desarrollo de competencias en programas de educación en línea que trabajan con la metodología de proyectos formativos Esto es clave para realizar posteriormente estudios experimentales. Con base en lo anterior, se establecieron dos objetivos para el presente estudio:

1. Identificar la utilidad de la metodología de proyectos formativos en el proceso de aprendizaje de los estudiantes y su rendimiento académico.

2. Determinar si la autoevaluación y la evaluación de los estudiantes se Corresponde con la hetero-evaluación del docente.

Al hablar de evaluación en las aulas siempre se debe tener en cuenta los procesos anteriormente mencionados puesto que permiten que el proceso se dé como un sistema más coherente y practico que desarrolla las necesidades, la evaluación en este caso se toma desde la reflexión social de la práctica vivida por los educandos estas permiten que se enumeren o se tengan en cuenta las cualidades o dificultades que se 
tienen para sí mismo y con ello la retroalimentación y la búsqueda de nuevas alternativas para poder generar reflexiones pertinentes a sus prácticas dando cuenta de todos los procesos a cambiar y así generar nuevas alternativas en el desarrollo de las praxis.

La educación se limita a solo recoger resultados cuantitativos y estos sean el indicador o parámetro final del resultado del contenido abordado traducido en aprendizaje. Hoy la perspectiva pretende superar y continuar avanzando a través de la evaluación socio-formativa la cual implica un cambio consiente y con resultados basados en experiencias colaborativas y auto-reflexivas con aspectos cualitativos y centrados en procesos de aprendizajes González, W. (2017).

Otra de las características se puede evidenciaren que requiere transformar el proceso de formación. Para ello, se propone la socio-formación, un enfoque que consiste en formar a las personas para que resuelvan problemas del contexto y contribuyan a la realización personal, la convivencia, el fortalecimiento del tejido social, el desarrollo socioeconómico y la sustentabilidad ambiental En la sociedad del conocimiento, cada vez más se promueve la participación activa del estudiante en el proceso de evaluación, lo cual es posible mediante diferentes tipos de evaluación. (Tobón, Pimienta y García, 2015).

Los contextos permiten desarrollar las experiencias para que las evaluaciones sean pertinentes, el desarrollo de habilidades y de estrategias en cada una de las actividades de la praxis son esenciales para reflexionar y demostrar cómo y conque el educando puede aportar a los conocimientos y de ahí hacer unas evaluaciones coherentes en el desarrollo de sus habilidades. También Generan nuevas alternativas de solución pedagógicas (Parra, 2006). Desde la socio-formación se propone el desarrollo de competencias sustentables en los diversos actores sociales (estudiantes, docentes, directivos, políticos, líderes, etc.), las cuales se refieren a actuaciones integrales para resolver problemas en situaciones complejas y cambiantes. Estas competencias se enfocan en generar nuevas alternativas de solución a los Problemas en situaciones reales, mediante proyectos de innovación e investigación, con apoyo en la colaboración inter, multi y trans disciplinaria. Implican saber conocer, saber hacer, saber convivir y saber ser, afrontando diferentes contingencias que pueden ser transferidas con creatividad a cualquier contexto actual y futuro.

Cabe resaltar desde los aportes anteriormente mencionados que también tomar a esta como un proceso de resolución de problemas (Tobón, 2012b) puesto que la docencia socio-formativa tiene las siguientes características principales Resolución de problemas: se abordan los problemas como retos para resolver necesidades, crear e innovar, y así contribuir a mejorar lo que se tiene (problemas del contexto). En este sentido son oportunidades para vivir mejor y no situaciones negativas que bloquean la actuación.

A comparación de la evaluación tradicional la evaluación socio formativa desarrolla al máximo las competencias a partir de problemas del contexto y el análisis de evidencias, estas diferencias con la evaluación según (Tobón,2015). Tradicionalmente la evaluación se ha enfocado para aprobar o reprobar a los alumnos en base a los resultados mostrados durante una prueba, una actividad, una secuencia, teniendo como consecuencia que el estudiante se sienta capaz en ese tema, área 
o curso, aunque no lo sea o por lo contrario, que se sienta frustrado, incapaz, no solo en el área que fue evaluado sino incluso en todos los ámbitos, lo que lleva a muchos estudiantes a abandonar sus estudios.

La evaluación es un constante sistema de medición de saberes donde la reflexión propia permite medir la información y la construcción de saberes que cada ser humano puede tener en su formación, de esta manera le permite al ser humano ser cambiante autónomo y practico en el desarrollo de sus conocimientos y en la difusión de lo que se quiere dar a conocer, en este caso la praxis se vuelve un proceso pertinente para medir o evaluar las capacidades que se logran y las necesidades que se deben mejorar o en su caso trabajar en ello.

Para Tobón (2015) la evaluación constructivista tiene como objeto los saberes en diferentes dimensiones: ser hacer, conocer y convivir; cuyo fin es determinar el aprendizaje de saberes. La metodología que presenta es análisis de saberes previos, abordaje de intereses de los estudiantes, autorregulación del estudiante, evaluación mediante situaciones con sentido o dinámicas. El papel del docente es acompañar y orientar a los estudiantes a que se autoevalúen y mejoren.

Constituye una respuesta con pertinencia, así como lo plantea Miranda (2014), la Educación Superior desde la socio-formación constituye una respuesta con pertinencia al encargo social, es un tema de reflexión de todas las instituciones universitarias debido a que durante mucho tiempo se ha formado profesionales centrados en los contenidos, enciclopedistas que poco o nada están preparados para enfrentar una práctica laboral y profesional. Siendo que hoy en día se requieren acciones educativas que respondan a la realidad en que vivimos y contribuyan a la formación de profesionales íntegros e integrales constituyendo de manera equilibrada los aspectos cognitivos, procedimentales, emocionales y éticos que son fundamentales para resolver los problemas de la sociedad y el vivir bien.

Para el enfoque socio-formativo, como lo plantean Tobón, Pimienta y García (2010) las competencias son actuaciones integrales ante actividades y problemas del contexto con idoneidad y compromiso ético, que integran el saber ser, el saber hacer y el saber conocer en una perspectiva de mejora continua. Una competencia entonces no es solo tener un saber hacer, un saber conocer o un saber ser por separado, sino movilizar los diversos saberes hacia el logro de una meta determinada en el contexto.

Por consiguiente, se confirma el hecho de que los maestros no deben asumir simplemente la adquisición de conocimientos por parte de los estudiantes sin la mediación del entendimiento, la comprensión, la reflexión, la postura crítica y por sobre todo la aplicación de ese conocer en un contexto determinado con responsabilidad social. En este mismo sentido el papel del maestro gira en torno a la reflexión sobre la pertinencia de los contenidos que enseña, acorde a las necesidades e interese de los estudiantes y sobre todo a que sean relevantes.

Los propósitos del estudio de caso fueron: 1) determinar el enfoque educativo o pedagógico seguido en la experiencia de evaluación analizada; 2) establecer los logros y áreas de oportunidad en la experiencia de evaluación teniendo en cuenta los referentes de la socioformación y los retos del desarrollo social sostenible con el apoyo en una rúbrica validada en el área; y 3) proponer acciones para mejorar la planeación de la evaluación y 
el diseño de los instrumentos empleados, con el fin de fortalecer la orientación hacia el abordaje de problemas y productos pertinentes en el contexto; y 4) sugerir algunos elementos para mejorar la implementación de la evaluación con base en la colaboración.

\section{Metodología}

\subsection{Tipo de Estudio}

Se siguió una metodología cualitativa, basada en el estudio de caso. El estudio de caso consiste en evaluar el curso proceso de investigación formativa $1^{\circ}$ y $2^{\circ}$ en cada una de sus estrategias metodológicas teóricas y prácticas para generar nuevas estrategias desde miradas reflexivas que contribuyen a mejoramientos en el ejercicio docente en el caso de quien lo orienta, y en la praxis en el caso de quien la ejecuta, el cual se caracteriza por precisar de un proceso de búsqueda e indagación, así como el análisis sistemático de uno o varios casos. Para ser más exactos, por caso entendemos todas aquellas circunstancias, situaciones o fenómenos únicos de los que se requiere más información o merecen algún tipo de interés dentro del mundo de la investigación.

\subsection{Experiencia Evaluada}

En la Tabla 1 En el Plan de Estudios del Programa de Pedagogía Infantil, el curso denominado "Proceso de Investigación Formativa: $1^{\circ}$ y $2^{\circ}$, en la Facultad de Ciencias de la Educación, tiene un fin académico, investigativo y de proyección social; se constituye en un Trabajo de Campo que realizan los estudiantes de VI semestre para profundizar lo aprendido en todos los cursos y en las etapas cursadas y aprobadas del Proceso de Investigación Formativa, de los semestres anteriores según las necesidades de formación de los estudiantes, pero en ningún momento para repetir lo actuado, -ialguien imagina una práctica en la cual el estudiante no adquiera conocimientos nuevos?- en instituciones de educación, o fundaciones de beneficio a la comunidad o de servicio social en donde se requiera de apoyo socio educativo.

Tabla 1. Características de la asignatura, en la cual se hizo el análisis del proceso de evaluación del aprendizaje.

\begin{tabular}{ll}
\hline $\begin{array}{l}\text { Asignatura o } \\
\text { módulo: } \\
\text { Semestre o } \\
\text { grado: }\end{array}$ & $\begin{array}{l}\text { Proceso de } \\
\text { formativa } 1^{\circ} \text { y } 2^{\circ}\end{array}$ \\
$\begin{array}{l}\text { Estructura de } \\
\text { la asignatura: }\end{array}$ & Teórico practica \\
$\begin{array}{l}\text { Modalidad: } \\
\text { Duración de la } \\
\text { asignatura en } \\
\text { horas: }\end{array}$ & $\begin{array}{l}\text { Presencial } \\
\text { Un semestre académico, con una } \\
\text { intensidad de ciento veintiocho } \\
\text { horas (128): treinta y dos (32) } \\
\text { horas teóricas y noventa y seis } \\
\text { (96) horas prácticas. }\end{array}$ \\
$\begin{array}{l}\text { Número de } \\
\text { créditos: }\end{array}$ & $\begin{array}{l}\text { Enfoque } \\
\text { pedagógico: }\end{array}$ \\
$\begin{array}{l}\text { Documentos } \\
\text { evaluados (solo } \\
\text { anotar las } \\
\text { referencias con } \\
\text { autor y año): }\end{array}$ & $\begin{array}{l}\text { Protocolo, diarios de campo, } \\
\text { auto-evaluaciones, valoraciones } \\
\text { de las jornadas, planeación, }\end{array}$ \\
\hline
\end{tabular}

Nota. La tabla muestra las diferentes características de la asignatura, en que se efectuó el análisis del proceso de evaluación del aprendizaje. Fuente: PEP (Licenciatura en Pedagogía Infantil, Plan 2006).

\subsection{Instrumento}

El diagnóstico en torno al proceso de evaluación en la asignatura del programa de licenciatura en pedagogía infantil.

Se llevó a cabo con base en la aplicación de la Rúbrica de Prácticas de Evaluación del 
Aprendizaje desde la Socio-formación (Tabla 2), la cual fue diseñada y validada por CIFE (2018). Este instrumento busca determinar el nivel de avance en la implementación de la evaluación orientada a la formación integral de personas para afrontar los retos de la sociedad del conocimiento.

Valora siete prácticas esenciales en la evaluación del aprendizaje, mediante cinco niveles de actuación en los cuales se aplica la taxonomía socioformativa (Tobón, 2018b): Nivel Muy Bajo (Preformal), Nivel Bajo (Receptivo), Nivel Medio (Resolutivo), Nivel Medio Alto (Autónomo) y Nivel Muy Alto (Estratégico). Esto se complementa mediante un análisis de logros y aspectos a mejorar en cada una de las prácticas.

Tabla 2. Estructura de la Rúbrica de Prácticas de Evaluación del Aprendizaje desde la Socioformación

\begin{tabular}{|c|c|}
\hline Indicador & Descripción \\
\hline $\begin{array}{c}1 . \\
\text { Integración } \\
\text { de la } \\
\text { evaluación } \\
\text { dentro de la } \\
\text { planeación } \\
\text { didáctica }\end{array}$ & $\begin{array}{l}\text { Consiste en la integración de la } \\
\text { evaluación diagnóstica, continua y } \\
\text { sumativa en la planeación didáctica, } \\
\text { mediante actividades orientadas a } \\
\text { los estudiantes, y basadas en la } \\
\text { resolución de problemas del } \\
\text { contexto y el logro de productos con } \\
\text { pertinencia en el entorno. Implica la } \\
\text { adecuación de las actividades a las } \\
\text { características de los alumnos con } \\
\text { base en un diagnóstico de éstos y de } \\
\text { sus intereses. }\end{array}$ \\
\hline $\begin{array}{c}2 . \\
\text { Evaluación } \\
\text { basada en } \\
\text { problemas } \\
\text { del contexto }\end{array}$ & $\begin{array}{l}\text { Se aborda la evaluación con base en } \\
\text { la identificación, comprensión, } \\
\text { argumentación y resolución de un } \\
\text { problema del contexto, } \\
\text { considerando el ciclo vital de los } \\
\text { alumnos. Los problemas se asumen } \\
\text { como retos que deben lograrse para } \\
\text { superar necesidades y contribuir así } \\
\text { al desarrollo social sostenible. Esto } \\
\text { implica la aplicación del análisis } \\
\text { crítico y la creatividad en la } \\
\text { evaluación. }\end{array}$ \\
\hline
\end{tabular}

\begin{tabular}{|c|c|}
\hline $\begin{array}{c}3 . \\
\text { Evaluación } \\
\text { con base en } \\
\text { productos } \\
\text { de } \\
\text { desempeño }\end{array}$ & $\begin{array}{l}\text { Se busca el logro de un producto } \\
\text { pertinente en el contexto, a partir de } \\
\text { la consideración de un problema o } \\
\text { reto, teniendo en cuenta los intereses } \\
\text { de los alumnos. El producto puede } \\
\text { ser tangible o intangible, e implica } \\
\text { tener en cuenta el proceso por medio } \\
\text { del cual se llega a éste. }\end{array}$ \\
\hline $\begin{array}{c}4 . \\
\text { Evaluación } \\
\text { con base en } \\
\text { instrumento } \\
\quad \mathrm{s}\end{array}$ & $\begin{array}{l}\text { Consiste en el empleo de } \\
\text { instrumentos para evaluar tanto el } \\
\text { proceso como el producto obtenido } \\
\text { por los alumnos, a medida que } \\
\text { abordan un problema del contexto. } \\
\text { Los instrumentos ante todo } \\
\text { enfatizan en la autoevaluación y } \\
\text { coevaluación, y son conocidos por } \\
\text { los estudiantes desde el inicio de las } \\
\text { actividades. Se tienen en cuenta las } \\
\text { sugerencias de los estudiantes para } \\
\text { mejorar los instrumentos, los cuales } \\
\text { también son revisados por colegas } \\
\text { docentes. Tienen adaptación a los } \\
\text { alumnos con alguna discapacidad o } \\
\text { a los procesos de inclusión. }\end{array}$ \\
\hline $\begin{array}{c}5 . \\
\text { Retroalime } \\
\text { ntación } \\
\text { integral }\end{array}$ & $\begin{array}{l}\text { Se trata de que los estudiantes } \\
\text { posean información clara, detallada } \\
\text { y oportuna en torno a sus logros y } \\
\text { áreas de oportunidad para que } \\
\text { implementen las mejoras necesarias } \\
\text { para conseguir los aprendizajes } \\
\text { esperados. Este proceso se } \\
\text { implementa con base en la } \\
\text { comunicación asertiva y se capacita } \\
\text { a los mismos estudiantes para que } \\
\text { retroalimenten de forma positiva a } \\
\text { sus compañeros. }\end{array}$ \\
\hline $\begin{array}{c}6 . \\
\text { Evaluación } \\
\text { informal }\end{array}$ & $\begin{array}{l}\text { Consiste en aplicar la evaluación en } \\
\text { cualquier momento y espacio para } \\
\text { que el estudiante mejore y tenga } \\
\text { cada vez más impacto en la } \\
\text { resolución de los problemas y la } \\
\text { obtención de los productos } \\
\text { esperados en el entorno, sin } \\
\text { necesidad de que esté planeada o se } \\
\text { tenga un determinado instrumento. } \\
\text { Se orienta no solo al aprendizaje, } \\
\text { sino también a la formación integral. }\end{array}$ \\
\hline $\begin{array}{c}7 . \\
\text { Evaluación } \\
\text { formativa }\end{array}$ & $\begin{array}{l}\text { Busca la formación de los } \\
\text { estudiantes como ciudadanos } \\
\text { comprometidos con la mejora de las } \\
\text { condiciones de vida, el } \\
\text { emprendimiento, la convivencia y el } \\
\text { cuidado del ambiente, a través de }\end{array}$ \\
\hline
\end{tabular}


Fuente: (CIFE, 2018).

diversas acciones de apoyo, no sólo del docente, sino también de los pares, de la familia y del contexto social, dentro de un marco de trabajo colaborativo. A los estudiantes se les brindan varias oportunidades dentro de la evaluación hasta que logren los aprendizajes esperados al menos en un nivel básico en una asignatura, módulo o proyecto formativo.

\section{Resultados}

En la Tabla 3 se presentan los resultados de la evaluación curricular del programa por cada indicador evaluado. Puede observarse lo siguiente:

Integración de la evaluación dentro de la planeación didáctica, La evaluación dentro del proceso del PIF siempre ha estado inmersa puesto que permite demostrar la realidad con la teoría a tratar en cada uno de los escenarios donde se interviene, esta es continúa orientadas a los estudiantes, y basadas en la resolución de problemas del contexto y el logro de productos con pertinencia en el entorno.

Evaluación basada en problemas del contexto Con base a la exploración de los contestos (identificados en la lectura y análisis de contexto) los estudiantes que cursan el PIF dan cuenta de la necesidad que enfrentan, así mismo se debe hacer una articulación realizando las diferentes actividades y planes de acción en las intervenciones, esto permite un acercamiento especial a las realidades en las que como docentes se enfrentan, cada uno de estos se analiza y trabajan en las clases teóricas para que por socializaciones y debates grupales nazcan las posibles soluciones.
Evaluación con base en productos de desempeño Este proceso los estudiantes del programa y en especial del curso organizaron de acuerdo a sus experiencias, entregan al final del proceso un informe final de las actividades y de las intervenciones realizadas así mismo una sistematización y una socialización de sus experiencias en los escenarios educativos.

Evaluación con base en instrumentos, Los instrumentos logran que el estudiante suministré la información necesaria en cuanto a la institución donde realiza su práctica estos instrumentos enfatizan en la autoevaluación y evaluación, y son conocidos por los estudiantes desde el inicio de las actividades, con ellos se pude obtener información que luego le son de importación en el desarrollo de sus actividades.

Retroalimentación integra, Al momento de realizar las observaciones y las interacciones los estudiantes pueden dar cuenta de cada una de las necesidades con las que los contextos cuentan y a os cuales deben enfrentarse de la misma manera la articulación de las actividades deben estar encaminadas a desarrollar aprendizajes en cada una de las situaciones presentadas sin descuidar los parámetros establecidos por el PIF.

Evaluación informal, Este proceso es constante se evidencia en cada uno de las intervenciones, donde los estudiantes aparte de realizar sus apreciaciones y dar a conocer sus experiencias diarias (registradas en los diarios de campo) realizan una auto evaluación que le permite reflexionar sobre su quehacer pedagógico, esto se complementa con su desempeño en el aula. 


\begin{abstract}
Evaluación formativa, Bajo las experiencias en cada una de las intervenciones de las estudiantes en los escenarios pueden demostrar cual es compromiso que tiene en cuanto praxis educativa desarrollando planes de estudio donde dan respuesta a necesidades según los contextos donde se ubican permitiendo ser reflexivo y analítico en cada una de sus intervenciones así logren los aprendizajes esperados al menos en un nivel ubicado.
\end{abstract}

Tabla 3. Sintesis de los resultados a partir de la aplicación de la rúbrica

\begin{tabular}{|c|c|c|}
\hline Indicador & Logros & $\begin{array}{l}\text { Aspectos por } \\
\text { mejorar }\end{array}$ \\
\hline $\begin{array}{c}\text { 1. Integración } \\
\text { de la } \\
\text { evaluación } \\
\text { dentro de la } \\
\text { planeación } \\
\text { didáctica }\end{array}$ & $\begin{array}{l}\text { Implica la } \\
\text { adecuación de } \\
\text { las actividades } \\
\text { a las } \\
\text { características } \\
\text { mediante } \\
\text { actividades de } \\
\text { los alumnos } \\
\text { con base en un } \\
\text { diagnóstico de } \\
\text { éstos y de sus } \\
\text { intereses. Estas } \\
\text { son pertinentes } \\
\text { en cada una de } \\
\text { las } \\
\text { interacciones y } \\
\text { desarrollan en } \\
\text { los estudiantes } \\
\text { la articulación } \\
\text { de actividades } \\
\text { la planificación } \\
\text { y la innovación } \\
\text { de estrategias } \\
\text { didácticas para } \\
\text { el acercamiento } \\
\text { de la realidad } \\
\text { con las bases } \\
\text { teóricas }\end{array}$ & $\begin{array}{l}\text { Las nuevas } \\
\text { indicaciones en } \\
\text { cuanto a las } \\
\text { planeaciones } \\
\text { de acuerdo a los } \\
\text { parámetros del } \\
\text { ministerio de } \\
\text { educación y las } \\
\text { exigencias de } \\
\text { las la } \\
\text { instituciones } \\
\text { con ello la } \\
\text { misma } \\
\text { articulación } \\
\text { didáctica y la } \\
\text { planificación } \\
\text { coherente de } \\
\text { actividades } \\
\text { acordes a las } \\
\text { edades y los } \\
\text { diferentes } \\
\text { niveles } \\
\text { formación, así de } \\
\text { como la } \\
\text { articulación de } \\
\text { los contextos y } \\
\text { las necesidades } \\
\text { existentes }\end{array}$ \\
\hline $\begin{array}{l}\text { 2. Evaluación } \\
\text { basada en } \\
\text { problemas del } \\
\text { contexto }\end{array}$ & $\begin{array}{l}\text { Se aborda la } \\
\text { evaluación con } \\
\text { base en la } \\
\text { identificación, }\end{array}$ & $\begin{array}{l}\text { Se plantea la } \\
\text { restructuración } \\
\text { de los } \\
\text { protocolos con }\end{array}$ \\
\hline
\end{tabular}

\begin{tabular}{|c|c|c|}
\hline & $\begin{array}{l}\text { comprensión, } \\
\text { argumentación } \\
\text { y resolución de } \\
\text { un problema } \\
\text { del contexto, } \\
\text { esto permite } \\
\text { que en cada una } \\
\text { de las } \\
\text { intervenciones } \\
\text { los estudiantes } \\
\text { logren mitigar } \\
\text { las falencias, } \\
\text { esto les permite } \\
\text { afianzar las } \\
\text { capacidades y } \\
\text { establecer rutas } \\
\text { que lleven a } \\
\text { mejoras en los } \\
\text { escenarios de } \\
\text { intervención }\end{array}$ & $\begin{array}{l}\text { el fin de } \\
\text { organizar y } \\
\text { replantear la } \\
\text { evaluación de } \\
\text { las } \\
\text { experiencias } \\
\text { vividas por los } \\
\text { estudiantes, } \\
\text { estos } \\
\text { contribuyen en } \\
\text { la actualización } \\
\text { de las } \\
\text { planeaciones y } \\
\text { las practicas }\end{array}$ \\
\hline $\begin{array}{l}\text { 3. Evaluación } \\
\text { con base en } \\
\text { productos de } \\
\text { desempeño }\end{array}$ & $\begin{array}{l}\text { Es pertinente el } \\
\text { proceso de } \\
\text { socialización } \\
\text { evidencia la } \\
\text { pertinencia de } \\
\text { la praxis en } \\
\text { cada uno de los } \\
\text { escenarios de } \\
\text { igual manera se } \\
\text { mide la } \\
\text { responsabilidad } \\
\text { y el } \\
\text { compromiso de } \\
\text { los estudiantes } \\
\text { en cada } \\
\text { práctica, esta } \\
\text { también } \\
\text { demuestra un } \\
\text { proceso } \\
\text { riguroso de } \\
\text { seguimiento y } \\
\text { pertinencia de } \\
\text { su identidad } \\
\text { profesional }\end{array}$ & $\begin{array}{l}\text { Se debe realizar } \\
\text { un ajuste a este } \\
\text { proceso de } \\
\text { evaluación de } \\
\text { los productos } \\
\text { teniendo como } \\
\text { base nuevos } \\
\text { aportes y } \\
\text { sistematización } \\
\text { que sean } \\
\text { significativos y } \\
\text { pertinentes a } \\
\text { cada una de sus } \\
\text { prácticas con } \\
\text { ello se } \\
\text { demuestra } \\
\text { también las } \\
\text { realidades y en } \\
\text { concordancia la } \\
\text { articulación } \\
\text { con los demás } \\
\text { cursos vistos } \\
\text { para que sea } \\
\text { más eficiente }\end{array}$ \\
\hline $\begin{array}{l}\text { 4. Evaluación } \\
\text { con base en } \\
\text { instrumentos }\end{array}$ & $\begin{array}{l}\text { Se tienen en } \\
\text { cuenta las } \\
\text { sugerencias de } \\
\text { los estudiantes } \\
\text { para mejorar } \\
\text { los } \\
\text { instrumentos, } \\
\text { puesto que } \\
\text { permiten un }\end{array}$ & $\begin{array}{l}\text { Establecer } \\
\text { algunas } \\
\text { mejoras donde } \\
\text { los } \\
\text { instrumentos } \\
\text { no se } \\
\text { conviertan en } \\
\text { un sistema } \\
\text { repetitivo y con }\end{array}$ \\
\hline
\end{tabular}




\begin{tabular}{|c|c|c|}
\hline & $\begin{array}{l}\text { mejor } \\
\text { desarrollo en la } \\
\text { adquisición de } \\
\text { conocimientos, } \\
\text { estos a su vez } \\
\text { son una guía } \\
\text { investigativa, } \\
\text { pero deben } \\
\text { estar centrados } \\
\text { en las } \\
\text { necesidades } \\
\text { que cada } \\
\text { contexto } \\
\text { presenta }\end{array}$ & $\begin{array}{l}\text { ello se pierden } \\
\text { las intenciones } \\
\text { de los mismo } \\
\text { cada nivel } \\
\text { cursado y cada } \\
\text { praxis lograda } \\
\text { determinan } \\
\text { diferentes } \\
\text { necesidades y } \\
\text { allí los } \\
\text { instrumentos } \\
\text { pueden proyectar estas } \\
\text { de manera } \\
\text { eficiente }\end{array}$ \\
\hline $\begin{array}{c}5 . \\
\text { Retroalimenta } \\
\text { ción integral }\end{array}$ & $\begin{array}{l}\text { Se trata de que } \\
\text { los estudiantes } \\
\text { posean } \\
\text { información } \\
\text { clara, detallada } \\
\text { y oportuna en } \\
\text { torno a sus } \\
\text { logros y áreas } \\
\text { de oportunidad, } \\
\text { las mejoras } \\
\text { necesarias para } \\
\text { conseguir los } \\
\text { aprendizajes } \\
\text { esperados. Este } \\
\text { proceso se } \\
\text { implementa } \\
\text { con base en la } \\
\text { comunicación } \\
\text { asertiva y se } \\
\text { capacita a los } \\
\text { mismos } \\
\text { estudiantes } \\
\text { para que } \\
\text { retroalimenten } \\
\text { de forma } \\
\text { positiva a sus } \\
\text { compañeros. }\end{array}$ & $\begin{array}{l}\text { Se deben } \\
\text { establecer en } \\
\text { los protocolos } \\
\text { diferentes } \\
\text { diagnósticos } \\
\text { donde los } \\
\text { estudiantes al } \\
\text { momento de } \\
\text { realizar su } \\
\text { praxis } \\
\text { evidencien } \\
\text { aparte de las } \\
\text { necesidades del } \\
\text { aula otras } \\
\text { dificultades } \\
\text { sociales y del } \\
\text { contexto que le } \\
\text { pueden servir } \\
\text { de anclaje a sus } \\
\text { actividades y } \\
\text { que el impacto } \\
\text { sea mayor }\end{array}$ \\
\hline $\begin{array}{l}\text { 6. Evaluación } \\
\text { informal }\end{array}$ & $\begin{array}{l}\text { Se ha podido } \\
\text { establecer las } \\
\text { categorías con } \\
\text { las cuales los } \\
\text { estudiantes } \\
\text { pueden } \\
\text { establecer sus } \\
\text { criterios de } \\
\text { autoevaluación } \\
\text { esto les permite } \\
\text { dar un giro u }\end{array}$ & $\begin{array}{lr}\text { Se debe } \\
\text { establecer una } \\
\text { nueva mirada a } \\
\text { la evaluación } \\
\text { donde se } \\
\text { involucren las } \\
\text { nuevas } \\
\text { relaciones e } \\
\text { interpretacione } \\
\text { s con relación } \\
\text { al proceso de }\end{array}$ \\
\hline
\end{tabular}

\begin{tabular}{|c|c|c|}
\hline & $\begin{array}{l}\text { organizar la } \\
\text { praxis } \\
\text { pedagógica, así } \\
\text { mismo la } \\
\text { evaluación en } \\
\text { el pif le permite } \\
\text { ser autocritico } \\
\text { de su labor }\end{array}$ & $\begin{array}{l}\text { evaluación } \\
\text { sumado a estas } \\
\text { la formación } \\
\text { socio formativa } \\
\text { que contribuye } \\
\text { en parte a la } \\
\text { caracterización } \\
\text { de nuevos } \\
\text { saberes }\end{array}$ \\
\hline $\begin{array}{l}\text { 7. Evaluación } \\
\text { formativa }\end{array}$ & $\begin{array}{lr}\text { Con la } & \text { evaluación } \\
\text { logra que el } \\
\text { educando sea } \\
\text { más eficiente y } \\
\text { busque de } \\
\text { manera } \\
\text { continua las } \\
\text { herramientas } \\
\text { necesarias para } \\
\text { dar una praxis } \\
\text { pertinente sin } \\
\text { dejar de } \\
\text { articular las } \\
\text { bases o } \\
\text { fundamentos } \\
\text { teóricos que le } \\
\text { aportan esas } \\
\text { estrategias } \\
\text { necesarias para } \\
\text { el desarrollo de } \\
\text { sus } \\
\text { intervenciones }\end{array}$ & $\begin{array}{lr}\text { Buscar la } & \text { la } \\
\text { necesidad de } & \text { los } \\
\text { que estudiantes } & \\
\text { sean más } \\
\text { autocríticos en } \\
\text { su ejercicio y } \\
\text { con ello la } \\
\text { práctica se } \\
\text { convierta en } \\
\text { ese proceso de } \\
\text { auto evaluación } \\
\text { que desarrolle } \\
\text { fines no solo de } \\
\text { impartir los } \\
\text { conocimientos } \\
\text { en la } \\
\text { escenarios, sino } \\
\text { que también le } \\
\text { den hen las } \\
\text { herramientas } \\
\text { para lograrlo }\end{array}$ \\
\hline
\end{tabular}

Fuente: Elaboración propia

\section{Discusiones}

En el análisis del caso expuesto, se puede observar que en la práctica evaluativa que se brinda en la asignatura de proceso de investigación formativa $1^{\circ}$ y $2^{\circ}$ prima; el enfoque cualitativo como énfasis, en los aspectos análisis y descripciones de las experiencias de las praxis en los escenarios educativos y como estas influyen de manera positiva o negativa en la formación de los licenciados, así mismo toma como referencia las necesidades que se presentan en el aula para dar una mirada evaluativa del mismo. 
La planeación de la evaluación, dentro de las actividades didácticas consiste en medir y contribuir en las actividades para el desarrollo de los procesos de enseñanza aprendizaje y es un elemento clave en el diseño del micro currículo, como las asignaturas, módulos o proyectos formativos.

En el presente estudio, se encontraron logros tales como: la adecuación de las actividades a las características mediante actividades de los alumnos con base en un diagnóstico de éstos y de sus intereses. Estas son pertinentes en cada una de las interacciones y desarrollan en los estudiantes la articulación de actividades la planificación y la innovación de estrategias didácticas para el acercamiento de la realidad con las bases teóricas.

Se aborda la evaluación con base en la identificación, comprensión, argumentación y resolución de un problema del contexto, esto permite que en cada una de las intervenciones los estudiantes logren mitigar las falencias, esto les permite afianzar las capacidades y establecer rutas que lleven a mejoras en los escenarios de intervención.

Es pertinente el proceso de socialización evidencia la pertinencia de la praxis en cada uno de los escenarios de igual manera se mide la responsabilidad y el compromiso de los estudiantes en cada práctica, esta también demuestra un proceso riguroso de seguimiento y pertinencia de su identidad profesional.

Se tienen en cuenta las sugerencias de los estudiantes para mejorar los instrumentos, puesto que permiten un mejor desarrollo en la adquisición de conocimientos, estos a su vez son una guía investigativa, pero deben estar centrados en las necesidades que cada contexto presenta.
Se trata de que los estudiantes posean información clara, detallada y oportuna en torno a sus logros y áreas de oportunidad, las mejoras necesarias para conseguir los aprendizajes esperados. Este proceso se implementa con base en la comunicación asertiva y se capacita a los mismos estudiantes para que retroalimenten de forma positiva a sus compañeros.

Se ha podido establecer las categorías con las cuales los estudiantes pueden establecer sus criterios de autoevaluación esto les permite dar un giro u organizar la praxis pedagógica, así mismo la evaluación en el PIF le permite ser autocritico de su labor.

Con la evaluación se logra que el educando sea más eficiente y busque de manera continua las herramientas necesarias para dar una praxis pertinente sin dejar de articular las bases o fundamentos teóricos que le aportan esas estrategias necesarias para el desarrollo de sus intervenciones y los aspectos a mejorar son Se debe realizar un ajuste a este proceso de evaluación de los productos teniendo como base nuevos aportes y sistematización que sean significativos y pertinentes a cada una de sus prácticas con ello se demuestra también las realidad y en concordancia la articulación con los demás cursos vistos para que sea más eficiente.

Establecer algunas mejoras donde los instrumentos no se conviertan en un sistema repetitivo y con ello se pierden las intenciones de los mismo cada nivel cursado y cada praxis lograda determinan diferentes necesidades y allí los instrumentos pueden proyectar estas de manera eficiente.

Se deben establecer en los protocolos diferentes diagnósticos donde los estudiantes al momento de realizar su praxis evidencien aparte de las necesidades del aula otras 
dificultades sociales y del contexto que le pueden servir de anclaje a sus actividades y que el impacto sea mayor.

Finalmente, se requiere establecer una nueva mirada a la evaluación donde se involucren las distintas relaciones e interpretaciones con relación al proceso de evaluación, sumado, la formación socio formativa que contribuye en parte a la caracterización de nuevos saberes.

\section{Conclusiones}

La evaluación basada en problemas del contexto es una característica central de la evaluación socio formativa y consiste en un proceso de diagnóstico, retroalimentación y apoyo continuo a las personas, equipos, organizaciones y comunidades para que aprendan a resolver problemas del contexto retadores, mejoren en su actuación y desarrollen el talento necesario para la sociedad del conocimiento.

En el presente estudio, se pudo determinar que se tienen avances en cada uno de los proceso de formación en cuanto al desarrollo de las practicas pero se requiere mejorar en ejes como resolver problemas del contexto retadores, mejoren en su actuación y desarrollen el talento necesario para la sociedad del conocimiento, mediante la autoevaluación, con evaluación y hetero evaluación, teniendo como base la elaboración de productos (evidencias) e indicadores (o instrumentos) que posibiliten la metacognición, a través del trabajo colaborativo y el pensamiento complejo. Respecto a la orientación de la evaluación con base en problemas del entorno, otros estudios reportan avances en el proceso, como es que no solo se presentan las evidencias, sino que cada una demanda una reflexión y análisis que permite a la persona autoevaluarse, conocer más a fondo su proceso de aprendizaje y encontrar sus debilidades, áreas de oportunidad y sus fortalezas; con ello se busca que posteriormente vaya en busca de la mejora (Vázquez, 2015).

La evaluación con base en productos de desempeño es otro eje de la evaluación socio formativa y consiste en los procesos donde los estudiantes del programa y en especial del curso organizaron de acuerdo a sus experiencias, entregan al final del proceso un informe final de las actividades y de las intervenciones realizadas así mismo una sistematización y una socialización de sus experiencias en los escenarios educativos Se caracteriza por que aborda la evaluación con base en la identificación, comprensión, argumentación y resolución de un problema del contexto, considerando el ciclo vital de los alumnos. Los problemas se asumen como retos que deben lograrse para superar necesidades y contribuir así al desarrollo social sostenible. Esto implica la aplicación del análisis crítico y la creatividad en la evaluación.

En todo proceso de evaluación de competencias los instrumentos son esenciales, pero esto debe hacerse con base en los siguientes elementos, contextos, estrategias características entre otros; En el estudio de caso realizado, se encontró q logran que el estudiante suministre la información necesaria en cuanto a la institución donde realiza su práctica estos instrumentos enfatizan en la autoevaluación y evaluación, y son conocidos por los estudiantes desde el inicio de las actividades. 


\section{Referencias}

Aguilar A. A. (2017). Concept and methodology of the socioformative evaluation. CIFE University Center.

CIFE (2018). Rubric of Learning Evaluation Practices from the Socioformation. CIFE

Hernández M. J. S., Tobón S., Guerrero Ro. G. (2016). Towards a comprehensive evaluation of performance: the socioformative rubrics. Indigenous Autonomous

Salazar-Gomez, E., \& Tobon, S. (2018). Documentary analysis of the teacher training process according to the knowledge society. Spaces, 39 (45), 17.

Tobón, S. (2013). Integral training and skills. Complex thinking, curriculum, teaching and evaluation. Bogotá: ECOE. http://cort.as/-N_9P

Tobón, S. (2017). Socioformative evaluation. Strategies and instruments. Mount

Dora (USA): Kresearch. ISBN 978-1945721-26 -7. Link: https://cife.edu.mx/recursos/2018/08/ 23/evaluacion-socioformativa/

Tobón, S. (2017). Essential axes of knowledge society and socioformation. Mount

Dora: Kresearch. DOI: dx.doi.org/10.24944/isbn. 978-1945721-19-9 link: https://cife.edu.mx/recursos/2018/09/ 03/essential-axes-of-knowledge-
society-and-socioformation/Spanish versión: https: /cife.edu.mx/recursos/2018/09/06/eje s-esenciales-de-la-sociedad-delconocimiento-y-la-socioformacion/

Tobón, S. (2018). Guide for curriculum design and redesign from socioformation and complex thinking. Mount Dora: Kresearch. Download from: https://cife.edu.mx/recursos/

Tobón, S., Martinez, J. E., Valdez, E., \& Quiriz, T. (2018). Pedagogical practices: Analysis through conceptual mapping. Espacios, 39 (45), 26. Download from: https://www.researchgate.net/publica tion/328851969_Practicas_pedagogic as_Analisis_mediante_la_cartografia _conceptual_Pedagogical_Practices_ Analysis_by_conceptual_cartography

Tobón, S., Pimienta-Prieto, J. H., \& GarcíaFraile, J. A. (2016). Didactic sequences and socioformation. Mexico City: Pearson.

Zabala, A., y Arnau, L. (2007). How to learn and teach skills. Barcelona: Graó. Recovered from http://goo.gl/bXkiYg

Zabalza, M.A. (2018). Learning Assessment. In S. R. Herrera-Meza and S. Tobón (Moderators), III International Evaluation Congress. Congress conducted by the CIFE University Center, Cuernavaca, Mexico. Link: https://cife.edu.mx/recursos/2018/10/ 03/evaluacion-del-aprendizajeenfoque-formativo/

Dora (USA): Kresearch. 98 p. ISBN: 978-1945721-26-7

Education Recovered:https://cife.edu.mx/recurs 
os/wp-

content/uploads/2018/08/LIBRO-

Evaluaci\%C3\%B3n-Socioformativa1.0-1.pdf

University of México (2016). El Fuerte, México Ra Ximhai, vol. 12, no. 6, pp. 359-376 Recovered:

http://www.redalyc.org/articulo.oa?id $=46148194025$ 